\title{
A Parameter Estimation Approach to State Observation of Nonlinear Systems ${ }^{\text {th }}$
}

\author{
Romeo Ortega $^{\mathrm{a}}$, Alexey Bobtsov ${ }^{\mathrm{b}}$, Anton Pyrkin $^{\mathrm{b}}$, Stanislav Aranovskiy $^{\mathrm{b}}$ \\ ${ }^{a}$ Laboratoire des Signaux et Systèmes, CNRS-SUPELEC, Plateau du Moulon, 91192, Gif-sur-Yvette, \\ France \\ ${ }^{b}$ Department of Control Systems and Informatics, ITMO University, Kronverkskiy av. 49, Saint \\ Petersburg, 197101, Russia
}

\begin{abstract}
A novel approach to the problem of partial state estimation of nonlinear systems is proposed. The main idea is to translate the state estimation problem into one of estimation of constant, unknown parameters related to the systems initial conditions. The class of systems for which the method is applicable is identified via two assumptions related to the transformability of the system into a suitable cascaded form and our ability to estimate the unknown parameters. The first condition involves the solvability of a partial differential equation while the second one requires some persistency of excitation-like conditions. The proposed observer is shown to be applicable to position estimation of a class of electromechanical systems, for the reconstruction of the state of power converters and for speed observation of a class of mechanical systems.
\end{abstract}

Keywords: Parameter estimation, Adaptive observer, Nonlinear systems

\section{Introduction}

The problem of designing observers for nonlinear systems has received a lot of attention due to its importance in practical applications, where some of the states may not be available for measurement. The interested reader is referred to [4, 5] for a recent review of the literature.

In this paper a new framework for constructing globally convergent (reduced-order) observers for a well-defined class of nonlinear systems is presented. Instrumental to this development is to formulate the observer design problem as a problem of parameter estimation, which represents the initial conditions of the unknown part of the state. This new family of observers are called parameter estimation-based observers (PEBO). The class of systems for which PEBO is applicable is identified via two assumptions. The first one characterizes, via the solvability of a partial differential equation (PDE), systems for which there exists a partial change of coordinates that assigns a particular cascaded structure to the system that permits to obtain a classical regression form involving only measurable quantities and the unknown parameter. The second assumption pertains to our ability to consistently estimate this unknown parameter that, in general, may enter nonlinearly in the regression form. For linear regression forms, which may be obtained via over-parameterisation of the nonlinear regression, many well-established parameter estimation algorithms are available and the

\footnotetext{
This paper is submitted to Systems \& Control Letters Journal. The abridged version of this paper will be presented at Conference on Descision and Control 2015, Osaka, Japan.
} 
second assumption can be replaced by the well-known persistency of excitation (PE) condition [20, 27]. The latter condition is related with the "regular input" or "universal input" assumptions imposed in standard observer designs [4, 5, 10,

It should be underscored that, in contrast with the classical observer design method based on linearization up to output injection [17, where the PDE to be solved imposes stringent conditions on the system, this is not the case for our PDE. The proposed PEBO also compares favourably with Kazantzis-Kravaris-Luenberger observers [15] in the following sense. Although both observers require an injectivity condition, in our observer this is imposed only on the partial change of coordinates mapping while in the Kazantzis-KravarisLuenberger observers the stronger requirement of injectivity of the full-state change of coordinates is needed. As is well-known [2] ensuring the latter injectivity property is the main stumbling block for the application of this kind of observers.

The method is shown to be applicable for position estimation of a class of electromechanical systems. This class contains, as a particular case, the interesting example of permanent magnet synchronous motors (PMSM) that have been widely studied in the control and drives literature - see [1, 25] and references therein. It also allows us to design observers for a class of power converters under more realistic measurement assumptions than the existing results obtained with other observer design techniques. Finally, it generates simple speed observers for mechanical systems that are partially linearisable via change of coordinates (PLvCC) - a practically important class that has been thoroughly studied in [29].

The remaining of the paper is organized as follows. Section 2 presents the problem formulation and main result. Section 3 is devoted to a discussion of the results. The case of linear time-invariant (LTI) systems is treated in Section 4 . Section 5 illustrates the application of the technique to three physical examples. The paper is wrapped-up with concluding remarks and future research directions in Section 6 .

\section{Problem Formulation and Main Result}

In this section the (partial state) observer problem addressed in the paper, and the approach that we propose to solve it, are presented. The class of systems for which the PEBO design technique is applicable is identified via two assumptions. The first one, given in Subsection 2.2, characterizes systems for which there exists a partial change of coordinates that assigns a particular cascaded structure to the system that permits to reformulate the state observation problem as a problem of parameter estimation. The second assumption, given in Subsection 2.3 , pertains to our ability to consistently estimate this unknown parameter.

\subsection{Partial state observer design problem}

Consider the dynamical system

$$
\begin{aligned}
& \dot{x}=f_{x}(x, y, u) \\
& \dot{y}=f_{y}(x, y, u),
\end{aligned}
$$

where $f_{x}: \mathbb{R}^{n_{x}} \times \mathbb{R}^{n_{y}} \times \mathbb{R}^{m} \rightarrow \mathbb{R}^{n_{x}}$ and $f_{y}: \mathbb{R}^{n_{x}} \times \mathbb{R}^{n_{y}} \times \mathbb{R}^{m} \rightarrow \mathbb{R}^{n_{y}}$ are smooth mappings ${ }^{1}$ Assume that the input signal vector $u: \mathbb{R}_{+} \rightarrow \mathbb{R}^{m}$ is such that all trajectories of the system are bounded. Find, if possible, mappings $F: \mathbb{R}^{n_{\xi}} \times \mathbb{R}^{n_{y}} \times \mathbb{R}^{m} \rightarrow \mathbb{R}^{n_{\xi}}$ and $G$ :

\footnotetext{
${ }^{1}$ Throughout the paper it is assumed that all mappings are sufficiently smooth.
} 
$\mathbb{R}^{n_{\xi}} \times \mathbb{R}^{n_{y}} \times \mathbb{R}^{m} \rightarrow \mathbb{R}^{n_{x}}$, for some positive integer $n_{\xi}$, such that the (partial state) observer

$$
\begin{aligned}
\dot{\xi} & =F(\xi, y, u) \\
\hat{x} & =G(\xi, y, u),
\end{aligned}
$$

ensures that $\xi$ is bounded and

$$
\lim _{t \rightarrow \infty}|\hat{x}(t)-x(t)|=0,
$$

for all initial conditions $(x(0), y(0), \xi(0)) \in \mathbb{R}^{n_{x}+n_{y}+n_{\xi}}$ and a well defined class of input signals $u \in \mathcal{U}$.

It is important to underscore that, in contrast with the usual observer problem formulation, a provision regarding the input signal is added. This additional qualifier is needed because the observation problem will be recast in terms of parameter estimation whose solution requires "sufficiently exciting" signals. See Section 1.2 in [5] for a thorough discussion of the role of the input in the observation problem. Also, note that we have writen the system dynamics including the output $y$ as part of the state, this is done, of course, without loss of generality,

\subsection{System re-parametrization}

Assumption 1. There exists three mappings

$$
\begin{aligned}
\phi & : \mathbb{R}^{n_{x}} \times \mathbb{R}^{n_{y}} \rightarrow \mathbb{R}^{n_{z}} \\
\phi^{\mathrm{L}} & : \mathbb{R}^{n_{z}} \times \mathbb{R}^{n_{y}} \rightarrow \mathbb{R}^{n_{x}} \\
h & : \mathbb{R}^{n_{y}} \times \mathbb{R}^{m} \rightarrow \mathbb{R}^{n_{z}},
\end{aligned}
$$

with $n_{z} \geq n_{x}$, verifying the following conditions.

(i) (Left invertibility of $\phi(\cdot, \cdot)$ with respect to its first argument)

$$
\phi^{\mathrm{L}}(\phi(x, y), y)=x, \quad \forall x \in \mathbb{R}^{n_{z}}, \forall y \in \mathbb{R}^{n_{y}} .
$$

(ii) (Transformability into cascade form)

$$
\frac{\partial \phi}{\partial x} f_{x}(x, y, u)+\frac{\partial \phi}{\partial y} f_{y}(x, y, u)=h(y, u)
$$

An immediate corollary of (ii) in Assumption 1 is that the partial change of coordinates

$$
z=\phi(x, y)
$$

ensures

$$
\dot{z}=h(y, u) .
$$

Moreover, the left invertibility condition (i) ensures that the partial state $x$ can be recovered from $z$ and $y$, that is,

$$
x=\phi^{\mathrm{L}}(z, y) .
$$

The cascade structure of the system is given in Fig. 1 . 


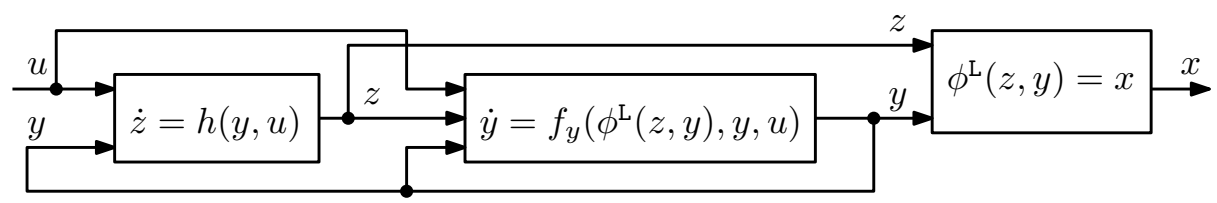

Figure 1: Block diagram representation of of the transformed system.

Proposition 1. Consider the system (1) verifying Assumption 1. Define the dynamic extension

$$
\dot{\chi}=h(y, u)
$$

with $\chi(0) \in \mathbb{R}^{n_{z}}$. We can compute a mapping $\Phi: \mathbb{R}^{n_{z}} \times \mathbb{R}^{n_{y}} \times \mathbb{R}^{m} \times \mathbb{R}^{n_{z}} \rightarrow \mathbb{R}^{n_{y}}$ such that

$$
\begin{aligned}
& \dot{y}=\Phi(\chi, y, u, \theta) \\
& x=\phi^{\mathrm{L}}(\chi+\theta, y),
\end{aligned}
$$

where $\theta \in \mathbb{R}^{n_{z}}$ is a vector of constant, unknown parameters.

Proof 1. From (6) and (8) we get $\dot{z}=\dot{\chi}$. Hence, integrating this equation yields

$$
z(t)=\chi(t)+\theta
$$

where

$$
\theta:=z(0)-\chi(0)
$$

Replacing (11) in (7) yields (10). Finally, the regression model (9) is obtained replacing (10) in (1) to get

$$
f_{y}\left(\phi^{\mathrm{L}}(\chi+\theta, y), y, u\right)=: \Phi(\chi, y, u, \theta)
$$

completing the proof.

\subsection{Consistent parameter estimation}

An immediate consequence of Proposition 1 is that the problem of observation of the unmeasurable state $x$ is translated into a standard parameter estimation problem for the regression model (9) with the observed state generated by

$$
\hat{x}=\phi^{\mathrm{L}}(\chi+\hat{\theta}, y)
$$

where $\hat{\theta}: \mathbb{R}_{+} \rightarrow \mathbb{R}^{n_{z}}$ is an on-line estimate of the constant vector $\theta$. Therefore, to complete the PEBO design it is necessary to ensure the existence of a consistent estimator for the unknown parameter $\theta$. Towards this end, the assumption below is introduced.

Assumption 2. There exists two mappings

$$
\begin{aligned}
& H: \mathbb{R}^{n_{z}} \times \mathbb{R}^{n_{\zeta}} \times \mathbb{R}^{n_{y}} \times \mathbb{R}^{m} \rightarrow \mathbb{R}^{n_{\zeta}} \\
& N: \mathbb{R}^{n_{z}} \times \mathbb{R}^{n_{\zeta}} \times \mathbb{R}^{n_{y}} \times \mathbb{R}^{m} \rightarrow \mathbb{R}^{n_{z}},
\end{aligned}
$$

with $n_{\zeta}>0$ such that the parameter estimator

$$
\begin{aligned}
& \dot{\zeta}=H(\chi, \zeta, y, u) \\
& \hat{\theta}=N(\chi, \zeta, y, u),
\end{aligned}
$$

coupled with the dynamic extension (8) and the regression model $(9)$ ensures that $\zeta$ is bounded and

$$
\lim _{t \rightarrow \infty}|\hat{\theta}(t)-\theta|=0,
$$

for all initial conditions $(y(0), \chi(0), \zeta(0)) \in \mathbb{R}^{n_{y}+n_{z}+n_{\zeta}}$ and a well defined class of input signals $u \in \mathcal{U}$. 


\subsection{Main result}

The main result of the paper is contained in the corollary below whose proof follows immediately from (8), (10), (14), (15) and the parameter convergence assumption (16).

Corollary 1. Consider the system (1) verifying Assumptions 1 and 2 with $u \in \mathcal{U}$. A (partial) state PEBO of the form (2) that guarantees (3) is given by

$$
\begin{aligned}
\xi & :=\operatorname{col}(\chi, \zeta) \\
F(\xi, y, u) & :=\left[\begin{array}{c}
h(y, u) \\
H(\chi, \zeta, y, u)
\end{array}\right] \\
G(\xi, y, u) & :=\phi^{\mathrm{L}}(\chi+N(\chi, \zeta, y, u), y) .
\end{aligned}
$$

\subsection{On the solvability of the PDE (4)}

Similarly to all constructive observer design methods the proposed technique involves the solution of a parameterised PDE, namely (4) - where we recall $h(y, u)$ is a free function. See [2, 4, 5, 10] for a recent review of the literature where the PDEs of the various existing observer design methods may be found. A key step in our observer design is, of course, the explicit solution of this PDE, some comments in this respect are made in this subsection.

To streamline the presentation of the subsequent discussion it is convenient to define the state vector $X:=\operatorname{col}(x, y)$ and the $(u$-parameterized) vector fields

$$
\begin{aligned}
f_{u}(X) & :=\operatorname{col}\left(f_{x}(X, u), f_{y}(X, u)\right) \\
h_{u}(X) & :=h(y, u)
\end{aligned}
$$

and rewrite the PDE (4) element-by-element as

$$
\frac{\partial \phi_{i}(X)}{\partial X} f_{u}(X)=\left(h_{u}\right)_{i}(X), \quad i=1, \ldots, n_{z} .
$$

Following the construction used in [8] we then define $n_{z}$ functions $\tilde{\phi}_{i}: \mathbb{R}^{n_{x}+n_{y}} \times \mathbb{R} \rightarrow \mathbb{R}$ as

$$
\tilde{\phi}_{i}(X, s):=\phi_{i}(X)-s, \quad i=1, \ldots, n_{z},
$$

and write the non-homogeneous PDE (18) as the following homogeneous PDE

$$
\frac{\partial \tilde{\phi}_{i}(X, s)}{\partial X} f_{u}(X)+\frac{\partial \tilde{\phi}_{i}(X, s)}{\partial s}\left(h_{u}\right)_{i}(X)=0 .
$$

Frobenius Theorem [13] states that, if

$$
\operatorname{rank}\left[\begin{array}{c}
f_{u}(X) \\
\left(h_{u}\right)_{i}(X)
\end{array}\right]=1, \quad i=1, \ldots, n_{z},
$$

(uniformly in $u$ ) in a neighbourhood of $X_{0} \in \mathbb{R}^{n_{x}+n_{y}}$, then 19 ) has a local solution around a point $\left(X_{0}, s_{0}\right) \in \mathbb{R}^{n_{x}+n_{y}} \times \mathbb{R}$, because all one-dimensional distributions are involutive. Unfortunately, the regularity condition (20) may be a restrictive assumption in the present scenario, since rules out solutions around equilibrium points of the system (1). This renders Frobenius Theorem unaplicable for our problem in - often encountered - stabilisation tasks. See Remark R5 in the next section.

Even in the case where the rank condition is satisfied it is clear that existence of solutions does not imply that an analytic expression for it can be easily obtained - and the requirement of solving the $\mathrm{PDE}$ remains the main stumbling block for our approach. 


\section{Discussion}

R1 Besides the explicit solution of the PDE (4) an additional difficulty is the selection of a mapping $h(y, u)$ that will ensure that the partial change of coordinates $\phi(x, y)$ admits a left inverse (with respect to $x$ ). It should be noted that this injectivity-like property is imposed on the partial change of coordinates $\phi(x, y)$, which should be contrasted with the requirement of injectivity of the full-state change of coordinates imposed in the KazantzisKravaris-Luenberger observers [15]. As discussed in [2] the latter injectivity property is the main stumbling block for the design of these observers - see [26] for a very illustrative example.

R2 For general nonlinear systems the regression system (9) depends nonlinearly on the unknown parameters. Although some results are available for the estimation of nonlinearly parameterised, nonlinear systems [3, 11, 18, 19, 28] the problem of generating consistent estimates remains wide open. On the other hand, for the case of linear parameterisation the estimation problem has a standard solution. Indeed, many techniques [4, 20, 27] are available to generate consistent estimates for linear regressions of the form

$$
\dot{y}=\Phi_{0}(\chi, y, u)+\Phi_{1}(\chi, y, u) \theta
$$

with known mappings $\Phi_{0}: \mathbb{R}^{n_{z}} \times \mathbb{R}^{n_{y}} \times \mathbb{R}^{m} \rightarrow \mathbb{R}^{n_{y}}$ and $\Phi_{1}: \mathbb{R}^{n_{z}} \times \mathbb{R}^{n_{y}} \times \mathbb{R}^{m} \rightarrow \mathbb{R}^{n_{y} \times n_{z}}$. See the examples in Section 5 .

R3 As is well-known [20], the parameter convergence requirement in parameter estimators involves some form of excitation on the signals - this requirement is encrypted in the condition $u \in \mathcal{U}$ of Assumption 2. This condition is of the same nature as the "universal input" or "regular input" conditions for classical observer designs [4, 5, 10]. For linear regressions of the form 21) it has a very precise characterisation in terms of $\mathrm{PE}$ of the regressor matrix $\Phi_{1}(\chi, y, u)$, which is defined as the existence of constants $\delta>0$ and $T>0$ such that for all $t \geq 0$

$$
\int_{t}^{t+T} \Phi_{1}^{\top}(\chi(s), y(s), u(s)) \Phi_{1}(\chi(s), y(s), u(s)) d s \geq \delta I_{n_{z}} .
$$

Under the PE condition above it is straightforward to design globally convergent parameter estimators for the regression model (21). In this case the set $\mathcal{U}$ is defined as follows:

$$
\mathcal{U}:=\left\{u: \mathbb{R}_{+} \rightarrow \mathbb{R}^{m} \mid(22) \text { holds along trajectories of (8), (9) }\right\} .
$$

From 222 it is clear that if there are more measured states than unknown ones, that is, if $n_{y} \geq n_{z}$, then the $\mathrm{PE}$ condition translates into a simple rank condition on the matrix $\Phi_{1}(\chi, y, u)$ - this is the case of mechanical systems treated in Subsection 5.1. It should be also recalled that in the identification literature there are well-known relationships between the adaptation gains, the PE constants $\delta$ and $T$ and the convergence rate of the estimation errors; see [21, 27].

R4 It should be underscored that, in many cases, it is possible to transform a nonlinearly parameterised regression into a linear one via over-parameterisation - see the discussion in this respect in [19]. Since over-parameterisation increases the dimension of the parameter space the excitation requirements on the signals are, of course, more stringent.

R5 A potential practical drawback of the proposed technique is the utilisation of pure integrators in (8). Indeed, in some applications the measurable signals may exhibit a (sign 
definite) bias in steady-state that will lead to unbounded signals when fed into open-loop integrators. On the other hand, this problem is conspicuous by its absence in regulation tasks. Indeed, in this case there exists a desired, constant operating point $\left(x_{*}, y_{*}\right) \in \mathbb{R}^{n_{x}+n_{y}}$ that must satisfy the equilibrium equations

$$
\begin{aligned}
& 0=f_{x}\left(x_{*}, y_{*}, u_{*}\right) \\
& 0=f_{y}\left(x_{*}, y_{*}, u_{*}\right),
\end{aligned}
$$

for some constant $u_{*} \in \mathbb{R}^{m}$. From the equations above it is clear that a necessary condition for solvability of the PDE (4) is that $h\left(y_{*}, u_{*}\right)=0$. Hence, in normal operating conditions, the open integration operation will not generate a bias. A similar scenario appears in the ubiquitous PI controllers widely used in industry to drive some error signal to zero. To avoid drift-e.g., in the presence of noise - several ad hoc remedies, including the addition of small leakages and resettings, are well established.

R6 As shown in (12) the unknown parameter $\theta$ - and, consequently, the estimated state - is determined by the system and observer initial conditions. It may be then argued that this makes our analysis "trajectory dependent", hence intrinsically fragile. This criticism would certainly be pertinent if off-line, instead of on-line, parameter estimators were advocated or if transient performance claims were made. Since this is not the case in the present work the argument seems specious.

\section{Case of Linear Time-Invariant Systems}

The proposed observer design procedure is of little - if at all-use for LTI systems. However, it is interesting to show that even for this simplest case the relationships between the classical notions of observability [14] and identifiability [30] and Assumptions 1 and 2 ] are far from obvious.

Proposition 2. Assume the system (1) is LTI, that is,

$$
\left[\begin{array}{c}
\dot{x} \\
\dot{y}
\end{array}\right]=\left[\begin{array}{ll}
A_{11} & A_{12} \\
A_{21} & A_{22}
\end{array}\right]\left[\begin{array}{l}
x \\
y
\end{array}\right]+\left[\begin{array}{l}
B_{1} \\
B_{2}
\end{array}\right] u
$$

where $A_{i j}$ and $B_{i}, i, j=1,2$, are constant matrices of suitable dimensions.

(C1) Observability of the system (23) (with respect to the output y) does not imply Assumption 1 .

(C2) Assumption 1 does not imply observability of the system (23).

(C3) If Assumption 1 holds then observability of the system (23) is necessary for identifiability [30] of the parameter $\theta$ (defined in Proposition 1). Hence, it is necessary for Assumption 2 to hold.

Proof 2. Without loss of generality we take

$$
\phi(x, y)=T_{1} x+T_{2} y,
$$

where $T_{1} \in \mathbb{R}^{n_{z} \times n_{x}}$ and $T_{2} \in \mathbb{R}^{n_{z} \times n_{y}}$. Since $n_{z} \geq n_{x}$, condition (i) of Assumption 1 is ensured imposing

$$
\operatorname{rank} T_{1}=n_{x}
$$


Under this conditions we have

$$
\phi^{\mathrm{L}}(z, y)=T_{1}^{\dagger}\left(z-T_{2} y\right)
$$

where

$$
T_{1}^{\dagger}:=\left(T_{1}^{\top} T_{1}\right)^{-1} T_{1}^{\top}
$$

The PDE (4) reduces to the algebraic equation

$$
T_{1} A_{11}+T_{2} A_{21}=0
$$

Now, applying Popov-Belevitch-Hautus criterion [14] we conclude that the system (23) is observable if and only if

$$
\operatorname{rank}\left[\begin{array}{c}
s I_{n_{x}}-A_{11} \\
A_{21}
\end{array}\right]=n_{x}, \quad \forall s \in \sigma\left\{A_{11}\right\}
$$

where $\sigma\{\cdot\}$ denotes the set of eigenvalues.

We prove claim $(\mathrm{C} 1)$ by constructing a system that is observable but does not satisfy Assumption 1. For, take $n_{x}=2$ and $n_{y}=1$ and set $A_{11}=\operatorname{diag}\left\{a_{1}, a_{2}\right\}$ with $a_{1} \neq a_{2} \neq 0$ and $A_{21}=\left[\begin{array}{ll}a & b\end{array}\right]$ with $a, b \neq 0$. The matrix appearing in (27) takes the form

$$
\left[\begin{array}{c}
s I_{2}-A_{11} \\
A_{21}
\end{array}\right]=\left[\begin{array}{cc}
s-a_{1} & 0 \\
0 & a-a_{2} \\
a & b
\end{array}\right],
$$

whose rank is two for $s=a_{i}, i=1,2$. Hence, the system is observable. On the other hand, for all $n_{z} \geq 2,26$ is equivalent to

$$
T_{1}=-T_{2} A_{21} A_{11}^{-1}
$$

Consequently

$$
\operatorname{rank} T_{1} \leq \min \left\{\operatorname{rank} T_{2}, \operatorname{rank} A_{21}\right\}=1<2=n_{x},
$$

violating the rank condition (24) .

Claim (C2) is proven by contradiction constructing a system that is not observable but satisfies Assumption 1. For, take $n_{x}=n_{y}=n_{z}=1$ and set $A_{21}=0$ that - from (27) implies the system is not observable. However, if $A_{11}=0$ the algebraic equation (26) admits a solution $T_{1}=1, T_{2}=0$ ensuring Assumption 1.

Finally, claim (C3) is established proving, after some lengthy but straightforward calculations, that the regression form $(9)$ is given by

$$
\dot{y}=A_{21} T_{1}^{\dagger} \chi+\left(A_{22}-A_{21} T_{1}^{\dagger} T_{2}\right) y+B_{2} u+A_{21} T_{1}^{\dagger} \theta,
$$

where we notice that $A_{21} T_{1}^{\dagger} \in \mathbb{R}^{n_{y} \times n_{z}}$ while $A_{21} \in \mathbb{R}^{n_{y} \times n_{x}}$. From [30] it follows that $\theta$ is identifiable if and only if $n_{y} \geq n_{z}$ and rank $A_{21}=n_{x}$-from the latter condition and (27) it is clear that observability follows.

\section{Application to Three Physical Examples}

In this section we prove that PEBO is applicable to the speed observation of PLvCC mechanical systems studied in [29], the position observation for a class of electromechanical systems, and the reconstruction of the full state from partial measurements of a popular switched power converter. 


\subsection{Mechanical systems which are partially linearisable via change of coordinates}

In this subsection we are interested in the problem of speed observation of mechanical systems described in Hamiltonian form by

$$
\left[\begin{array}{c}
\dot{y} \\
\dot{x}
\end{array}\right]=\left[\begin{array}{cc}
0 & I_{s} \\
-I_{s} & 0
\end{array}\right]\left[\begin{array}{c}
\frac{\partial H}{\partial y} \\
\frac{\partial H}{\partial x}
\end{array}\right]+\left[\begin{array}{c}
0 \\
G(y)
\end{array}\right] u
$$

where $s:=\frac{n}{2}$ is the number of degrees of freedom of the system, $y, x \in \mathbb{R}^{s}$ are the generalised position and momenta, respectively, $u \in \mathbb{R}^{m}$ is the control input, $m \leq s, G: \mathbb{R}^{s} \rightarrow \mathbb{R}^{s \times m}$ is the full rank input matrix. The Hamiltonian function $H: \mathbb{R}^{s} \times \mathbb{R}^{s} \rightarrow \mathbb{R}$ is the energy function

$$
H(y, x)=\frac{1}{2} x^{\top} M^{-1}(y) x+\mathcal{V}(y),
$$

where $M: \mathbb{R}^{s} \rightarrow \mathbb{R}^{s \times s}$ is the positive definite inertia matrix and $\mathcal{V}: \mathbb{R}^{s} \rightarrow \mathbb{R}$ is the potential energy function. It is assumed that position $y$ is measurable and we want to estimate velocity $\dot{y}$ via the estimation of momenta $x$ and the relation $\dot{y}=M^{-1}(y) x$.

It will be shown that if the dynamics can be rendered linear in momenta (velocities) via a change of coordinates then Assumptions 1 and 2 of the paper are satisfied. Moreover, the resulting reparameterisation is linear, that is, of the form (21), and the PE condition (22) is trivially satisfied, hence the set $\mathcal{U}$ is the whole input space. These systems, referred as PLvCC, have been studied in [29] and they have been characterised via the solvability of a PDE - see also [7] for an intrinsic characterisation of the class.

To present our result we need to recall the following assumption from [29].

Assumption 3. Given the inertia matrix $M(y)$. There exists a full rank matrix $\mathcal{T}: \mathbb{R}^{s} \rightarrow$ $\mathbb{R}^{s \times s}$ such that, for $i=1, \ldots, s$,

$$
\mathcal{B}_{(i)}(y)+\mathcal{B}_{(i)}^{\top}(y)=0,
$$

where the matrices $\mathcal{B}_{(i)}: \mathbb{R}^{s} \rightarrow \mathbb{R}^{s \times s}$ are defined as

$$
\mathcal{B}_{(i)}(y):=\sum_{j=1}^{n}\left\{\left[\mathcal{T}_{i}, \mathcal{T}_{j}\right] \mathcal{T}_{j}^{\top}\left(M \mathcal{T} \mathcal{T}^{\top}\right)^{-1}+\frac{1}{2} \mathcal{T}_{j i} \mathcal{T} \frac{\partial}{\partial q_{j}}\left(\mathcal{T}^{\top} M \mathcal{T}\right)^{-1} \mathcal{T}^{\top}\right\}
$$

with $\mathcal{T}_{i}(y)$ the $i$-th column of $\mathcal{T}(y), \mathcal{T}_{i j}(y)$ its $i j$-th element and $\left[\mathcal{T}_{i}, \mathcal{T}_{j}\right]$ the standard Lie bracket ${ }^{2}$ In this case the mechanical system $(28)$ is PLvCC.

Proposition 3. Consider the mechanical system (28) whose inertia matrix verifies Assumption 3 .

(i) Assumption 1 is satisfied with the mappings

$$
\begin{aligned}
\phi(x, y) & =\mathcal{T}^{\top}(y) x \\
\phi^{\mathrm{L}}(z, y) & =\mathcal{T}^{-\top}(y) z \\
h(y, u) & =-\mathcal{T}^{\top}(y)\left[\frac{\partial \mathcal{V}}{\partial y}(y)-G(y) u\right] .
\end{aligned}
$$

\footnotetext{
${ }^{2} \mathrm{~A}$ standard Lie Bracket of two vector fields $\mathcal{T}_{i}(y), \mathcal{T}_{j}(y)$ is defined as $\left[\mathcal{T}_{i}, \mathcal{T}_{j}\right]:=\frac{\partial \mathcal{T}_{j}}{\partial y} \mathcal{T}_{i}-\frac{\partial \mathcal{T}_{i}}{\partial y} \mathcal{T}_{j}$.
} 
(ii) The mapping $\Phi(\chi, y, u, \theta)$ of Proposition 1 is linear in $\theta$ and yields

$$
\dot{y}=\left[\mathcal{T}^{\top}(y) M(y)\right]^{-1}(\chi+\theta) .
$$

(iii) The PE condition (22) is satisfied for all input signals $u$. Hence, Assumption 2 holds with $\mathcal{U}$ being the whole input space.

Proof 3. Invoking Proposition 1 of [29] we have that the partial change of coordinates

$$
z=\mathcal{T}^{\top}(y) x
$$

transforms the system into the form

$$
\begin{aligned}
\dot{y} & =\left[\mathcal{T}^{\top}(y) M(y)\right]^{-1} z \\
\dot{z} & =-\mathcal{T}^{\top}(y)\left[\frac{\partial \mathcal{V}}{\partial y}(y)-G(y) u\right],
\end{aligned}
$$

which establishes claims (i) and (ii). To prove claim (iii) we refer to (21) and (31) and identify

$$
\Phi_{1}(\chi, y):=\left[\mathcal{T}^{\top}(y) M(y)\right]^{-1}
$$

which is a square, full rank matrix, hence 22 is trivially satisfied.

From Proposition 3 the design of a globally exponentially stable momenta observer follows trivially, and is omitted for brevity. It should be underscored that the resulting observer is much simpler than the I\&I observer proposed in [29]. This is particularly true for systems that do not satisfy the integrability Assumption 2 in [29], for which it is necessary to use the, rather involved, and high-gain-like technique of I\&I with dynamic scaling.

\subsection{Position observation in electromechanical systems}

We consider electromechanical system $3^{3}$ consisting of $n_{\lambda}$ inductances and a single mass, whose flux and mechanical position are denoted by $\lambda \in \mathbb{R}^{n_{\lambda}}$ and $q \in \mathbb{R}$, respectively. The magnetic energy stored in the inductances is given by

$$
\mathcal{E}_{\mathrm{M}}(i, q):=\frac{1}{2} i^{\top} L(q) i+\mu^{\top}(q) i
$$

where $L: \mathbb{R} \rightarrow \mathbb{R}^{n_{\lambda} \times n_{\lambda}}$ is the positive definite, (position-dependent) inductance matrix, $\mu: \mathbb{R} \rightarrow \mathbb{R}^{n_{\lambda}}$ represents the flux linkages due to the possible presence of permanent magnets and $i \in \mathbb{R}^{n_{\lambda}}$ are the currents flowing through the inductances. The kinetic energy of the mass is

$$
\mathcal{K}(\dot{q}):=\frac{1}{2} j \dot{q}^{2}
$$

with $j>0$ the (constant) mass inertia. We assume that the system is subject to constant external forces, e.g., gravitational forces, but it does not have any other potential energy storing elements. Hence, the systems potential energy is given by

$$
\mathcal{V}(q):=q \tau
$$

where $\tau \in \mathbb{R}$ represents the constant external force.

\footnotetext{
${ }^{3}$ The interested reader is referred to [22, 24] for additional details on this model.
} 
The dynamical model of the system is obtained applying Euler-Lagrange equations with the energy functions above yielding

$$
\begin{aligned}
L(q) \frac{d i}{d t}+L^{\prime}(q) \dot{q} i+\mu^{\prime}(q) \dot{q}+R i & =B u \\
j \ddot{q}-\frac{1}{2} i^{\top} L^{\prime}(q) i-i^{\top} \mu^{\prime}(q)+f \dot{q} & =-\tau,
\end{aligned}
$$

where $(\cdot)^{\prime}$ denotes differentiation, $R=\operatorname{diag}\left\{r_{1}, \ldots, r_{n_{\lambda}}\right\} \geq 0$ is the matrix of resistors (in series with the inductors), $B \in \mathbb{R}^{n_{\lambda} \times m}$ is a constant input matrix, $u \in \mathbb{R}^{m}$ are external voltage sources and $f \geq 0$ is a Coulomb friction coefficient.

As shown in [24 the model (32), (33) describes the behaviour of a large class of electromechanical systems, including the classical levitated ball and the most common electrical motors.

We are interested in the design of an observer for the mechanical position $q$ measuring $\operatorname{col}(i, \dot{q})$, as well as the more practically interesting case when we measure only $i$. To proceed with the observer design we recall Gauss's and Ampere's laws that establish the following expression for flux linkage vector

$$
\lambda=L(q) i+\mu(q)
$$

Moreover, Gauss's law tells us that

$$
\dot{\lambda}=-R i+B u
$$

The latter two equations correspond, of course, to the electrical equation (32). Since $i$ is measurable (35) proves that $L(q) i+\mu(q)$ qualifies as an admissible partial change of coordinates $4(x, y)$ verifying condition (ii) of Assumption 1 with $n_{z}=2$ and

$$
h(y, u):=-R i+B u .
$$

Condition (i) of Assumption 1 is satisfied if - given (34) - we can recover $q$ from measurement of $\lambda$ and $i$. Interestingly, we show below that this is the case for PMSMs.

Unfortunately, if the only measurable quantity is $i$, its time derivative $\frac{d i}{d t}$ in $(32)$ is not in the form of (1), that is $\dot{y}=f_{y}(x, y, u)$, with $x$ being only $q$ and $y$ only $i$. Indeed, besides $q$ and $i$, the electrical equation (32) contains the velocity $\dot{q}$. Hence, in order to obtain the regressor from (9) it is necessary to assume also measurement of $\dot{q}$.

Let us proceed now with the observer design for the (surface mount) PMSM [16, 24]. For the sake of clarity of exposition assume first that $i$ and $\dot{q}$ are measured - the requirement of measuring $\dot{q}$ is relaxed later. For the PMSM we have $n_{\lambda}=2, m=2, B=I_{2}$ and

$$
\begin{aligned}
L(q) & =L I_{2} \\
\mu(q) & =\lambda_{m}\left[\begin{array}{c}
\cos \left(n_{p} q\right) \\
\sin \left(n_{p} q\right)
\end{array}\right]
\end{aligned}
$$

where the positive constants $L, \lambda_{m}$ and $n_{p}$ are the stator inductance, permanent magnet flux constant and number of pole pairs, respectively. Hence, defining

$$
\phi(q, i):=L i+\lambda_{m}\left[\begin{array}{c}
\cos \left(n_{p} q\right) \\
\sin \left(n_{p} q\right)
\end{array}\right]=\lambda,
$$

\footnotetext{
${ }^{4}$ Notice that, with respect to the notation in Proposition $11 \lambda$ plays the role of $z$ and $q$ is $x$.
} 
it is clear that the mapping

$$
\phi^{\mathrm{L}}(\lambda, i):=\frac{1}{n_{p}} \arctan \left(\frac{\lambda_{2}-L i_{2}}{\lambda_{1}-L i_{1}}\right),
$$

satisfies condition (i) of Assumption 1 .

The dynamic extension (8) is given by

$$
\dot{\chi}=-R i+u .
$$

Now, from (35) (with $B=I_{2}$ ) and (37) we have, upon integration, that

$$
\lambda(t)=\chi(t)+\theta,
$$

where $\theta:=\lambda(0)-\chi(0)$ is the unknown parameter. After some lengthy, but straightforward calculations mimicking the proof of Proposition 1, we obtain a linear regression form for the currents as

$$
\frac{d}{d t}\left[\begin{array}{l}
i_{1} \\
i_{2}
\end{array}\right]=\Phi_{0}(\chi, i, \dot{q}, u)+\Phi_{1}(\dot{q}) \theta
$$

where

$$
\begin{aligned}
\Phi_{0}(\chi, i, \dot{q}, u) & :=\left[\begin{array}{cc}
-\frac{R}{L} & -n_{p} \dot{q} \\
n_{p} \dot{q} & -\frac{R}{L}
\end{array}\right]\left[\begin{array}{l}
i_{1} \\
i_{2}
\end{array}\right]+n_{p} \dot{q}\left[\begin{array}{l}
\chi_{2} \\
\chi_{1}
\end{array}\right]+u \\
\Phi_{1}(\dot{q}) & :=n_{p} \dot{q}\left[\begin{array}{l}
\chi_{2} \\
\chi_{1}
\end{array}\right]
\end{aligned}
$$

Although the regression form $(39)$ can be extended with an equation for $\ddot{q}$ this turns out to be unnecessary to solve the parameter estimation task that consists only of two unknown parameters.

A classical parameter estimator can be designed for the linear regression form (39). However, we make the important observation that the requirement of measuring $\dot{q}$, which is not realistic in a practical scenario, can be obviated. Towards this end we proceed as follow. First, from (34) and (36) we have that

$$
|\lambda-L i|^{2}=\lambda_{m}^{2}
$$

Second, replacing (38) above and expanding the square yields the static linear regression form

$$
Y(\chi, i)=S^{\top}(\chi, i) \eta
$$

where

$$
\begin{aligned}
Y(\chi, i) & :=|\chi-L i|^{2} \\
S(\chi, i) & :=\left[\begin{array}{c}
-2(\chi-L i) \\
1
\end{array}\right]
\end{aligned}
$$

are, of course, measurable and the new (extended) unknown parameter is

$$
\eta:=\left[\begin{array}{c}
\theta \\
\lambda_{m}^{2}-|\theta|^{2}
\end{array}\right]
$$

A full theoretical analysis and extensive simulations and experimental results of parameter estimators for the regressions (39) and (a filtered version of) (40) may be found in [6]. As shown in that paper the set $\mathcal{U}$ is defined as follows:

$$
\mathcal{U}:=\left\{u: \mathbb{R}_{+} \rightarrow \mathbb{R}^{2} \mid \int_{t}^{t+T} \dot{q}^{2}(s) d s \geq \delta>0, \text { along trajectories of (32), (33) and (36) }\right\} .
$$




\subsection{Cuk converter}

In this subsection we apply the proposed observer design technique to power converters. As will become clear below the technique applies to a broad class of converters, including the popular boost converter. For the sake of ease of exposition, instead of developing a - notationally cumbersome - general theory for a broader class of power converters, we preferred to concentrate on the specific example of the Cuk power converter, depicted in Fig. 2,

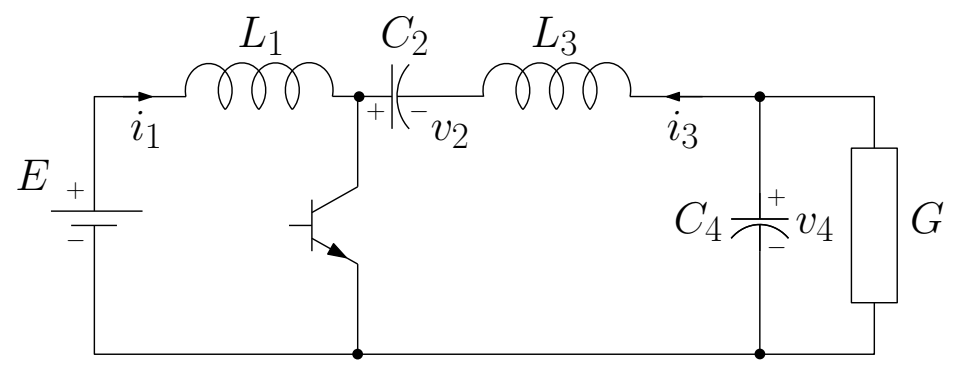

Figure 2: DC-DC Ćuk converter circuit

The average model of this device is given by

$$
\begin{aligned}
& L_{1} \frac{d i_{1}}{d t}=-(1-u) v_{2}+E \\
& C_{2} \dot{v}_{2}=(1-u) i_{1}+u i_{3} \\
& L_{3} \frac{d i_{3}}{d t}=-u v_{2}-v_{4} \\
& C_{4} \dot{v}_{4}=i_{3}-G v_{4}
\end{aligned}
$$

where $L_{1}, C_{2}, L_{3}, C_{4}, E$ and $G$ are positive constants and $u \in(0,1)$ is a duty cycle. We refer the reader to 4 for further details on the model.

To illustrate the generality of the approach we consider two different measurement scenarios. In the first one we assume that $\left(v_{2}, v_{4}\right)$ are measurable, while in the second one $\left(v_{2}, i_{3}\right)$ are measurable. Although from the practical viewpoint it is "easier" to measure voltages, we also consider the second one since, as shown in [4], is the one that can be solved with immersion and invariance (I\&I) observers, with which we compare our observer in simulations below.

Case I Denoting $y:=\operatorname{col}\left(v_{2}, v_{4}\right), x:=\operatorname{col}\left(i_{1}, i_{3}\right)$ we get from 41)

$$
\begin{aligned}
& \dot{x}_{1}=-\frac{1}{L_{1}}(1-u) y_{1}+\frac{E}{L_{1}} \\
& \dot{x}_{2}=-\frac{1}{L_{3}} u y_{1}-\frac{1}{L_{3}} y_{2} .
\end{aligned}
$$

Since the right hand side of these equations is independent of $x$ we can directly select

$$
\phi(x, y)=x .
$$

The dynamic extension is given by

$$
\dot{\chi}=\left[\begin{array}{c}
-\frac{1}{L_{1}}(1-u) y_{1}+\frac{E}{L_{1}} \\
-\frac{1}{L_{3}} u y_{1}-\frac{1}{L_{3}} y_{2}
\end{array}\right]=: h(y, u),
$$

and the regression form is

$$
\begin{aligned}
\dot{y} & =\Phi_{0}(\chi, y, u)+\Phi_{1}(u) \theta \\
\theta & :=x(0)-\chi(0)
\end{aligned}
$$


where

$$
\Phi_{0}(\chi, y, u):=\left[\begin{array}{c}
\frac{1}{C_{2}}(1-u) \chi_{1}+\frac{1}{C_{2}} u \chi_{2} \\
\frac{1}{C_{4}} \chi_{2}-\frac{G}{C_{4}} y_{2}
\end{array}\right], \Phi_{1}(u):=\left[\begin{array}{cc}
\frac{1}{C_{2}}(1-u) & \frac{1}{C_{2}} u \\
0 & \frac{1}{C_{4}}
\end{array}\right] .
$$

The model (42) contains the time derivative of the output $y$. To get a classical (static) regression model we use the standard filtering technique [23] and define the filtered signals

$$
\overline{(\cdot)}:=\frac{\alpha}{p+\alpha}(\cdot)
$$

where $p:=\frac{d}{d t}$ and $\alpha>0$ is a design parameter. Applying the filter to 42 we obtain the standard linear, static regression model

$$
\vartheta=\bar{\Phi}_{1} \theta+\epsilon
$$

where

$$
\vartheta:=\frac{\alpha p}{p+\alpha} y-\frac{\alpha}{p+\alpha} \Phi_{0}
$$

is clearly measurable (without differentiation) and $\epsilon$ is an exponentially decaying signal that depends on the filter initial conditions and the filter time constant $\frac{1}{\alpha}$.

The regression model (44) is used for the parameter estimator, which is the classical gradient estimator

$$
\dot{\hat{\theta}}=\Gamma \bar{\Phi}_{1}^{\top}\left(\vartheta-\bar{\Phi}_{1} \hat{\theta}\right)
$$

where the adaptation gain $\Gamma=\Gamma^{\top}>0$ is a design parameter. The state observer is defined as $\hat{x}=\hat{\theta}+\chi$.

It is important to underscore that the regressor matrix $\Phi_{1}(u)$ given in $(43)$ has an extremely simple form. Indeed, due to its upper triangular form, the estimation of the second parameter is decoupled from the first one and, moreover, the corresponding term in the regression is simply the constant $\frac{1}{C_{4}}$. Also, since the matrix depends only on the input signal $u$ the set $\mathcal{U}$ is defined as

$$
\mathcal{U}:=\left\{u: \mathbb{R}_{+} \rightarrow(0,1) \mid \int_{t}^{t+T}\left[\begin{array}{cc}
1-u(s) & (1-u(s)) u(s) \\
(1-u(s)) u(s) & u^{2}(s)+\frac{C_{2}^{2}}{C_{4}^{2}}
\end{array}\right] d s \geq \delta I_{2}>0\right\}
$$

Some simple calculations show that the matrix inside the integral is positive definite for any $u \in(0,1)$. Hence, $\mathcal{U}=\left\{u: \mathbb{R}_{+} \rightarrow(0,1)\right\}$ and consistent estimation is always guaranteed.

Simulations were carried out to evaluate the performance of the proposed observer. The simulations were done for the model (41) in closed-loop with the certainty equivalent version of the full-state feedback I\&I controller given in Proposition 8.2 of [4]. That is, the control law was defined by

$$
u=\frac{\left|V_{d}\right|}{\left|V_{d}\right|+E}+\lambda \frac{G\left|V_{d}\right| v_{2}+E\left(\hat{x}_{2}-\hat{x}_{1}\right)}{1+\left(G\left|V_{d}\right| v_{2}+E\left(\hat{x}_{2}-\hat{x}_{1}\right)\right)^{2}}
$$

where $V_{d}<0$ is the reference imposed to the output voltage $v_{4}$ and $\lambda$ is chosen as

$$
\lambda=\lambda_{0} \min \left(\frac{\left|V_{d}\right|}{\left|V_{d}\right|+E}, \frac{E}{\left|V_{d}\right|+E}\right),
$$

with $0<\lambda_{0}<2$. The full-state version of this controller, i.e., replacing $\hat{x}_{1}$ and $\hat{x}_{2}$ by $i_{1}$ and $i_{3}$, respectively, ensures global asymptotic stability of the desired equilibrium as well as verification of the saturation constraints in the input signal. 
The numerical simulations were performed with the following values of the converter parameters $L_{1}=10 \mathrm{mH}, C_{2}=22.0 \mu \mathrm{F}, L_{3}=10 \mathrm{mH}$ and $C_{4}=22.9 \mu \mathrm{F}, G=0.0447 \mathrm{~S}$ and $E=12 \mathrm{~V}$. The initial conditions for all simulations are set to $x(0)=(0.5,-1)$, $y(0)=(10,-12)$. The initial set point for the output voltage is $V_{d}=25 \mathrm{~V}$, and then this is changed at $t=0.2 \mathrm{~s}$ to $V_{d}=30 \mathrm{~V}$, at $t=0.4 \mathrm{~s}$ to $V_{d}=15 \mathrm{~V}$, at $t=0.6$ s to $V_{d}=5 \mathrm{~V}$, at $t=0.8 \mathrm{~s}$ to $V_{d}=20 \mathrm{~V}$. The simulation results are presented in Fig. 3 .

Case II Denoting now $y:=\operatorname{col}\left(v_{2}, i_{3}\right), x:=\operatorname{col}\left(i_{1}, v_{4}\right)$ we get from (41)

$$
\begin{aligned}
& \dot{x}_{1}=-\frac{1}{L_{1}}(1-u) y_{1}+\frac{E}{L_{1}} \\
& \dot{x}_{2}=\frac{1}{C_{4}} y_{2}-\frac{G}{C_{4}} x_{2} .
\end{aligned}
$$

The right hand side of the second equation depends on $x_{2}$, therefore the choice $\phi(x, y)=x$ is not suitable here. We propose instead

$$
\phi(x, y)=x-\left[\begin{array}{c}
0 \\
\frac{G L_{3}}{C_{4}} y_{2}
\end{array}\right]
$$

that, introducing the partial change of coordinates $z=\phi(x, y)$, yields the required form

$$
\dot{z}=\left[\begin{array}{c}
-\frac{1}{L_{1}}(1-u) y_{1}+\frac{E}{L_{1}} \\
\frac{1}{C_{4}} y_{2}+\frac{G}{C_{4}} u y_{1}
\end{array}\right]=: h(y, u) .
$$

The dynamic extension is then given by $\dot{\chi}=h(y, u)$, and the regression model is of the form

$$
\begin{aligned}
\dot{y} & =\Phi_{0}(\chi, y, u)+\Phi_{1}(u) \theta \\
\theta & :=x(0)-\chi(0)-\left[\begin{array}{c}
0 \\
\frac{G L_{3}}{C_{4}} y_{2}(0)
\end{array}\right],
\end{aligned}
$$

where

$$
\Phi_{0}(\chi, y, u):=\left[\begin{array}{c}
\frac{1}{C_{2}}(1-u) \chi_{1}+\frac{1}{C_{2}} u y_{2} \\
-\frac{1}{L_{3}} u y_{1}-\frac{1}{L_{3}} \chi_{2}-\frac{G L_{3}}{C_{4}} y_{2}
\end{array}\right], \Phi_{1}(u):=\left[\begin{array}{cc}
\frac{1}{C_{2}}(1-u) & 0 \\
0 & -\frac{1}{L_{3}}
\end{array}\right]
$$

The state observer takes the form

$$
\hat{x}=\hat{\theta}+\chi+\left[\begin{array}{c}
0 \\
\frac{G L_{3}}{C_{4}} y_{2}
\end{array}\right] .
$$

The regressor matrix $\Phi_{1}(u)$ given in 46 has an even simpler form than the one of Case I above. Indeed, the matrix is now diagonal with the second term in the regression simply the constant $\frac{-1}{L_{3}}$. Clearly, for this case we also have $\mathcal{U}=\left\{u: \mathbb{R}_{+} \rightarrow(0,1)\right\}$ and consistent estimation is always guaranteed.

In Proposition 8.3 of [4] the following I\&I observer is proposed

$$
\begin{aligned}
\hat{x}_{I \& I} & =\left[\begin{array}{l}
\zeta_{1} \\
\zeta_{2}
\end{array}\right]+\left[\begin{array}{l}
C_{2} \gamma_{1} y_{1} \\
L_{3} \gamma_{2} y_{2}
\end{array}\right] \\
\dot{\hat{\zeta}}_{1} & =\frac{1}{L_{1}}\left[-(1-u) y_{1}+E\right]-\gamma_{1}\left[(1-u)\left(\hat{\zeta}_{1}+C_{2} \gamma_{1} y_{1}\right)+u y_{2}\right] \\
\dot{\hat{\zeta}}_{2} & =\frac{1}{C_{4}}\left[y_{2}-G\left(\hat{\zeta}_{2}-L_{3} \gamma_{2} y_{2}\right)\right]-\gamma_{2}\left[u y_{1}+\hat{\zeta}_{2}-L_{3} \gamma_{2} y_{2}\right],
\end{aligned}
$$


where $\gamma_{1}, \gamma_{2}>0$ are design parameters. It should be noted that in the latter reference the parameters $E$ and $G$ are treated as unknown and are also estimated. If they are assumed known the I\&I observer takes the form given above.

The performance of our observer was compared with the I\&I observer (47) via numerical simulations. They were done under the same scenario as the ones done for Case I, but now with the certainty equivalent observer that results replacing $i_{1}$ and $v_{4}$ by $\hat{x}_{1}$ and $\hat{x}_{2}$, respectively. The simulation results are presented in Figs. 4,5 with different observer gains.

\section{Conclusions}

A radically new approach to design state observers for nonlinear systems has been proposed. The key idea is to translate the state observation problem into one of parameter estimation. It turns out that this is possible if we can find a partial change of coordinates $z=\phi(x, y)$ such that $\dot{z}$ depends only on $y$ and $u$. The observer then comprises a copy of $\dot{z}$ (called $\dot{\chi}$ ) that, upon integration, differs from $z$ only on the initial conditions - and these are the (constant) parameters that we propose to estimate. If the change of coordinates satisfies an injectivity property then $x$ can be estimated from the knowledge of $y, u, \chi$ and an estimate of $\theta$. Clearly, if the latter converges to $\theta$, then the estimate of $x$ will converge to its true value.

It has been shown in the paper that the change of coordinates is obtained from the solution of a parameterised PDE, which does not impose (a priori) the strict constraints of the classical observer design [17]. Moreover, it is argued that the required injectivity property is weaker than the one required in the Kazantzis-Kravaris-Luenberger and the I\&I observers.

The design of the observer is completed adding a parameter estimator to a regression model of the form $\dot{y}=\Phi(\chi, y, u, \theta)$ that, in general, depends nonlinearly on the parameters. Although some estimation techniques for nonlinearly parameterised nonlinear systems are available, it is also suggested that - via over-parameterisation-it may be possible to transform the regression into a linearly parameterised one. The latter case has been widely studied in the literature and many algorithms that guarantee parameter convergence under some excitation conditions are available.

The proposed technique has been shown to be applicable to position estimation of a class of electromechanical systems, to power converters and to speed estimation of the PLvCC mechanical systems studied in [29].

Current research is under way in the following directions.

- Identify other classes of physical systems to which the proposed method is applicable.

- Compare the performance of PEBO for the PLvCC mechanical systems discussed in Subsection 5.1 with other existing speed observers.

- Further clarify under which conditions the required partial change of coordinates exists and when it will lead to an easily tractable, linearly parameterised, regression model.

- Further explore the connection between the classical concepts of observability and identifiability and Assumption 1 and the excitation conditions required by the method. In this respect, the analysis of the simplest LTI case of Section 4 shows that this task is far from obvious. 


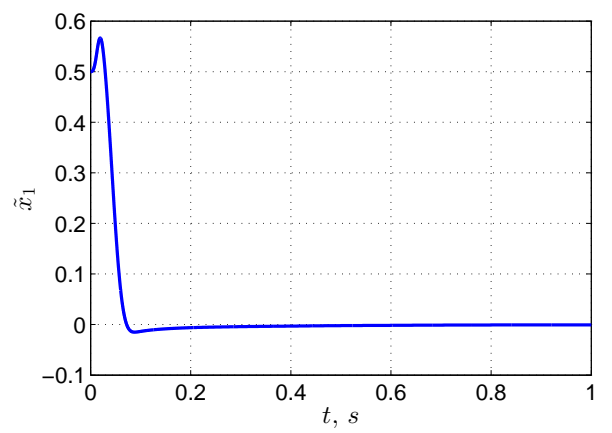

(a)

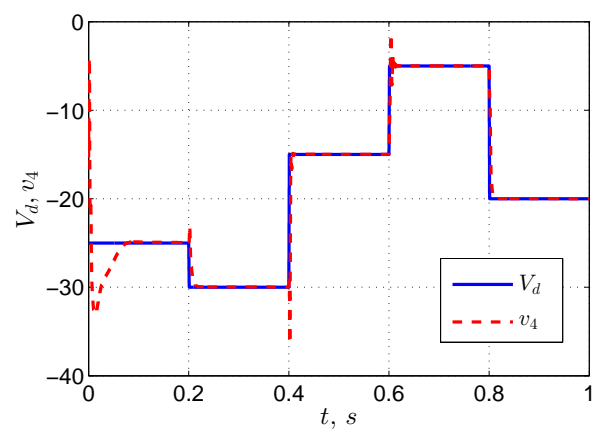

(c)

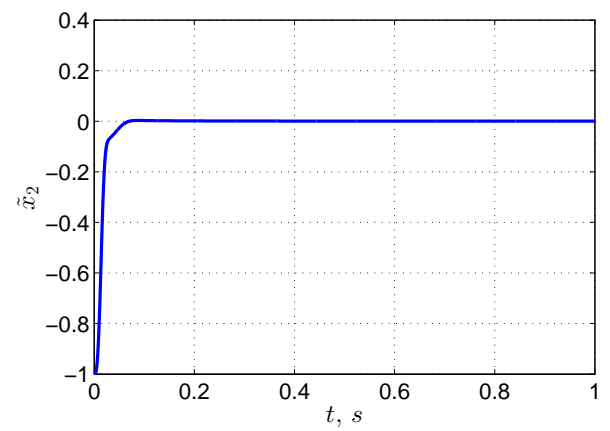

(b)

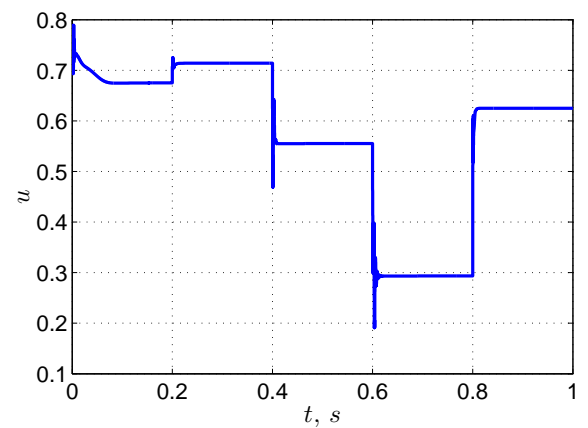

(d)

Figure 3: Transients of the observation errors (a) $\tilde{x}_{1}:=\hat{x}_{1}-i_{1}$, (b) $\tilde{x}_{2}:=\hat{x}_{2}-i_{3}$, (c) the voltage reference $V_{d}$ and voltage output $v_{4}$ and (d) the control input $u$ for the tuning gains $\alpha=0.5, \Gamma=0.001 I_{2}$. 


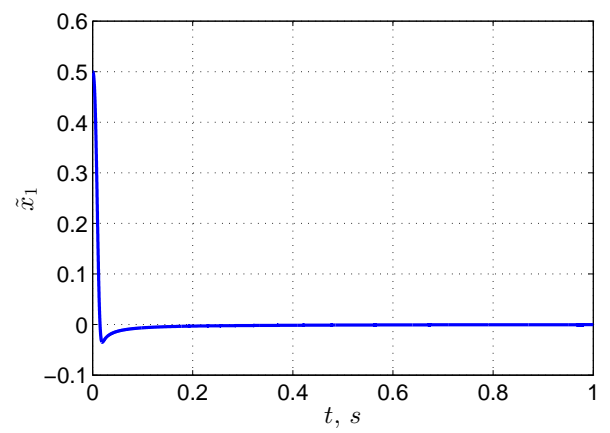

(a)

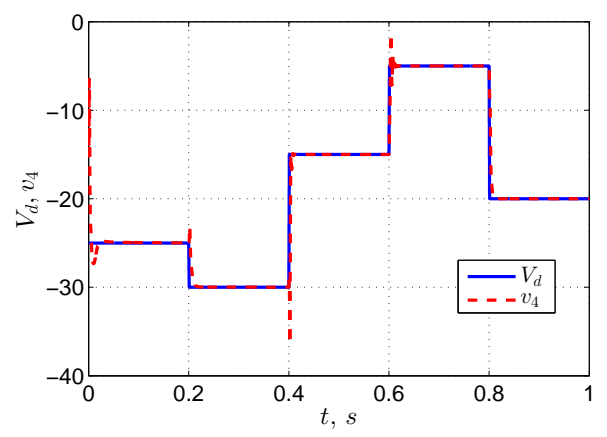

(c)

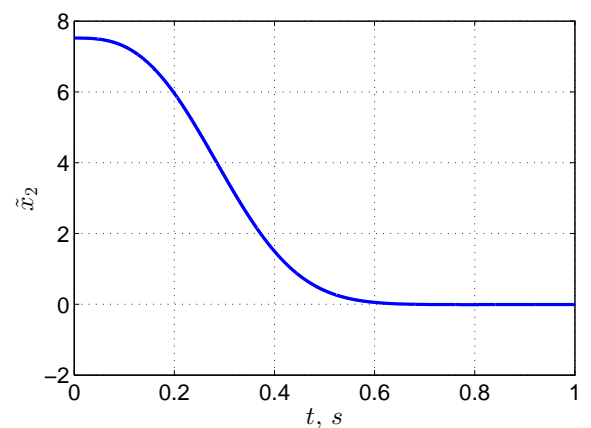

(b)

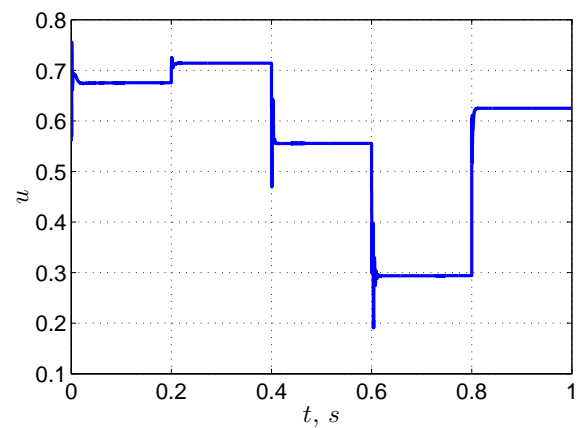

(d)

Figure 4: Transients of the observation errors (a) $\tilde{x}_{1}:=\hat{x}_{1}-i_{1}$, (b) $\tilde{x}_{2}:=\hat{x}_{2}-v_{4}$, (c) the voltage reference $V_{d}$ and voltage output $v_{4}$ and (d) the control input $u$ for the proposed observer with tuning gains $\alpha=1, \Gamma=\operatorname{diag}\{0.01,0.1\}$. 


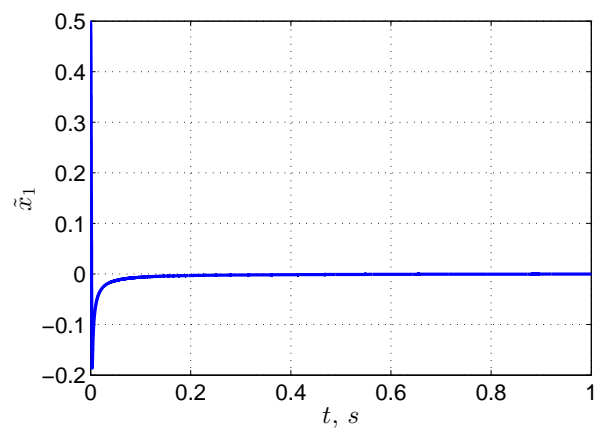

(a)

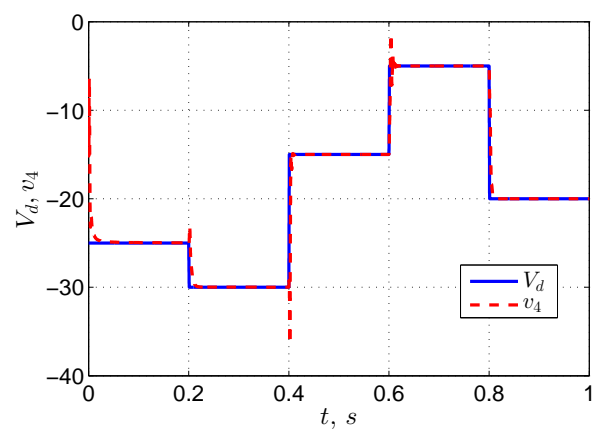

(c)

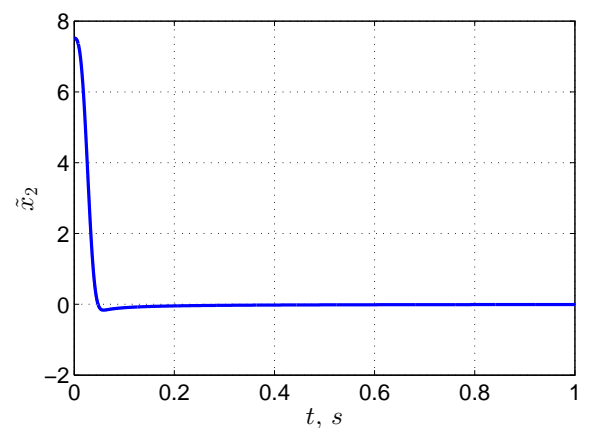

(b)

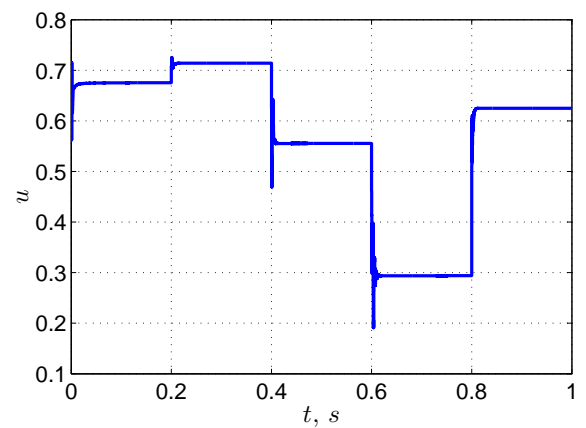

(d)

Figure 5: Transients of the observation errors (a) $\tilde{x}_{1}:=\hat{x}_{1}-i_{1}$, (b) $\tilde{x}_{2}:=\hat{x}_{2}-v_{4}$, (c) the voltage reference $V_{d}$ and voltage output $v_{4}$ and (d) the control input $u$ for the proposed observer with tuning gains $\alpha=1, \Gamma=\operatorname{diag}\{1,10\}$. 


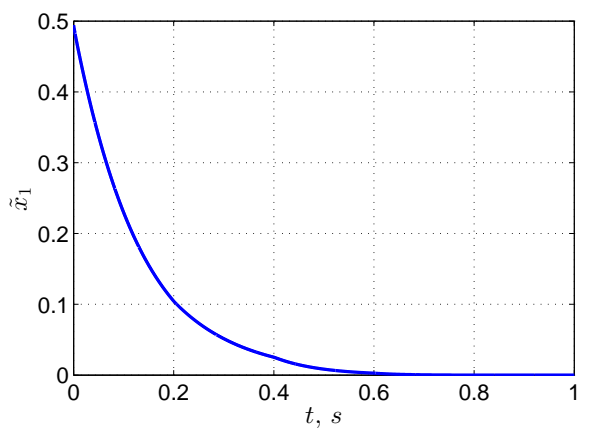

(a)

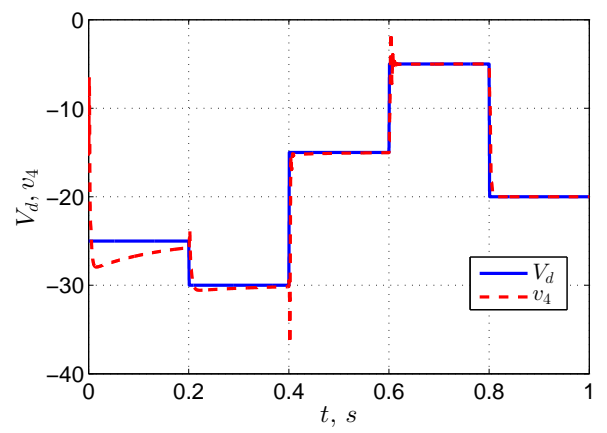

(c)

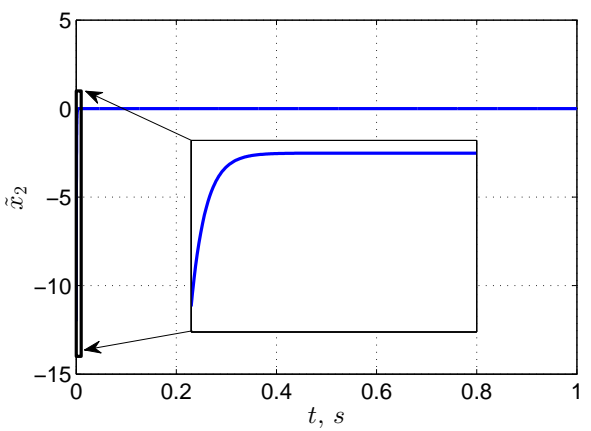

(b)

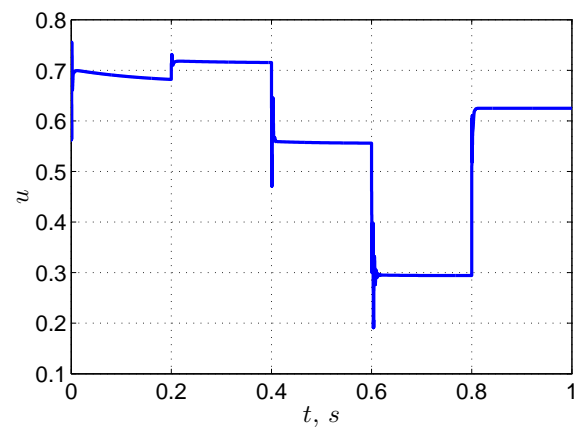

(d)

Figure 6: Transients of the observation errors (a) $\tilde{x}_{1}$, (b) $\tilde{x}_{2}$, (c) the voltage reference $V_{d}$ and voltage output $v_{4}$ and (d) the control input $u$ for the I\&I observer 47) with tuning gains $\gamma_{1}=25, \gamma_{2}=1$. 


\section{Acknowledgement}

The first author thanks Laurent Praly for some important clarifications on Assumption 1 and Vincent Andrieu for pointing out an error regarding the solvability of the PDE (4) found in a previous version of the paper.

This article is supported by the Ministry of Education and Science of Russian Federation (project 14.Z50.31.0031).

\section{References}

[1] P.P. Acarnley, J.F. Watson, Review of position-sensorless operation of brushless permanent-magnet machines, IEEE Transactions on Industrial Electronics, 2006, 53(2): 352-362.

[2] V. Andrieu, L. Praly, On the Existence of a Kazantzis-Kravaris/Luenberger Observer, SIAM Journal on Control and Optimization, 2006, 45(2): 432-456.

[3] A. Annaswamy, F. Skantze and A. Loh, Adaptive control of continuous time systems with convex/concave parametrization, Automatica, vol. 34, no. 1, pp. 33-48, 1998.

[4] A. Astolfi, D. Karagiannis and R. Ortega, Nonlinear and Adaptive Control with Applications, Springer-Verlag, Berlin, Communications and Control Engineering, 2007.

[5] G. Besançon (Ed.), Nonlinear Observers and Applications, Lecture Notes in Control and Information Science, Vol. 363, Springer-Verlag, 2007.

[6] A. Bobtsov, A. Pyrkin, and R. Ortega, A new approach for estimation of electrical parameters and flux observation of permanent magnet synchronous motors, nt. J. Adapt. Control Signal Process, 2015.

[7] D. Chang, R. G. McLenaghan, Geometric criteria for the quasi-linearization of the equations of motion of mechanical systems, IEEE Trans. Automat. Contr., vol. 58, No. 4, pp 1046-1050, 2013.

[8] D. Cheng, A. Astolfi and R. Ortega, On feedback equivalence to port-controlled Hamiltonian systems, Systems and Control Letters, Vol. 4, No. 9, pp 911-918, 2005.

[9] BMP Synchronous motor, Motor manual, V1.00, 12.2012, Schneider Electric, 2012.

[10] J. P Gauthier and T. Kupka, Deterministic Observation Theory and Applications Cambridge University Press, 2001.

[11] H. Grip, A. Saberi and T. Johansen, Estimation of states and parameters for linear systems with nonlinearly parameterized perturbations, Systems and Control Letters, vol. 60 , no. 9 , pp. $771-777,2011$.

[12] P. Ioannou and J. Sun, Robust Adaptive Control, PTR Prentice-Hall, 1996.

[13] A. Isidori, Nonlinear control systems (Vol. 1), Springer Science \& Business Media, 1995.

[14] T. Kailath, Linear Systems, Englewood Cliffs, NJ: Prentice-Hall, 1980.

[15] N. Kazantzis, C. Kravaris, Nonlinear observer design using Lyapunov?s auxiliary theorem. Systems \& Control Letters, 1998, 34(5): 241-247. 
[16] P.C. Krause, Analysis of Electric Machinery, McGraw Hill, New York, 1986.

[17] A.J. Krener and W. Respondek, Nonlinear observers with linearisable error dynamics, SIAM J. Control and Optimization, Vol. 23, No. 2, March 1985, pp. 197-217.

[18] X. Liu, R. Ortega, H. Su, and J. Chu, Immersion and invariance adaptive control of nonlinearly parameterized nonlinear systems, IEEE Trans. Automat. Contr., vol. 55, no. 9, pp. 2209-2214, 2010.

[19] X. Liu, R. Ortega, H. Su, and J. Chu, On adaptive control of nonlinearly parameterized nonlinear systems: Towards a constructive procedure, Systems \& Control Letters, vol. 60, pp. 36-43, 2011.

[20] L. Ljung, Systems Identification: Theory for the User, Prentice Hall, 1987.

[21] A. Loria, Explicit convergence rates for MRAC-type systems, Automatica, 40 (8), 14651468, 2004.

[22] J. Meisel, Principles of Electromechanical Energy Conversion, Krieger Pub Co, 1984.

[23] R.H. Middleton, G.C. Goodwin, Adaptive computed torque control for rigid link manipulations, Systems \& Control Letters, 1988, 10(1): 9-16.

[24] R. Ortega, A. Loria, P. J. Nicklasson and H. Sira-Ramirez, Passivity-Based Control of Euler-Lagrange Systems, Springer-Verlag, Berlin, Communications and Control Engineering, 1998.

[25] R. Ortega, L. Praly, A. Astolfi, J. Lee, K. Nam, Estimation of rotor position and speed of permanent magnet synchronous motors with guaranteed stability, IEEE Transactions on Control Systems Technology, 2011, 19(3): 601-614.

[26] L. Praly , L. Marconi and A. Isidori, A new observer for an unknown harmonic oscillator, In Proceedings of the 17th International Symposium on Mathematical Theory of Networks and Systems, Kyoto, Japan, 2006, July, pp. 24-28.

[27] S. Sastry, M. Bodson. Adaptive Control: Stability, Convergence and Robustness, Prentice Hall, Englewood Cliffs, NJ, 1989.

[28] I. Y. Tyukin, D. V. Prokhorov and C. V. Leeuwen, Adaptation and parameter estimation in systems with unstable target dynamics and nonlinear parametrization, IEEE Trans. Automat. Contr., vol. 52, no. 9, pp. 1543-1559, Sep. 2007.

[29] A. Venkatraman, R. Ortega, I. Sarras and A. van der Schaft, Speed observation and position feedback stabilization of partially linearizable mechanical systems, IEEE Transactions on Automatic Control, 55(5); 1059-1074, 2010.

[30] E. Walter, Identifiability of Parametric Models, Pergamon Press, Inc., 1987. 